\title{
ERGONOMÍA E INCLUSIÓN LABORAL DE PERSONAS CON DISCAPACIDAD INTELECTUAL LEVE. FEBRERO DE 2019
}

\section{ERGONOMICS AND LABOR INCLUSION OF PEOPLE WITH MILD INTELLECTUAL DISABILITY. FEBRUARY 2019}

\author{
Diana Yulieth Castañeda Zapata, Jessica María Carvajal Pardo, Adrián Augusto Leal Aranguren, Luz América \\ Martínez Álvarez y Cecilia Andrea Ordóñez Hernández \\ Semillero de Investigación en Ergonomía-SERGOS \\ Grupo de Investigación en Salud, Ambiente y Productividad-GISAP \\ Institución Universitaria Antonio José Camacho \\ Grupo de Investigación ESCULAPIO \\ Universidad Libre \\ Recibido: 07/04/2020. Aprobado: 29/04/2021 \\ Cómo citar este artículo: \\ Castañeda Zapata, D.Y., Carvajal Pardo, J.M., Leal Aranguren, A.A., Martínez Álvarez, L.A. y Ordóñez Hernández, C.A. \\ (2021). Ergonomía e inclusión laboral de personas con discapacidad intelectual leve. Febrero de 2019. Revista Sapientía, 13 (25), 6 - 15.
}

\section{RESUMEN}

Actualmente la discapacidad es reconocida como un tema fundamental de derechos humanos, ya que la mayoría de las personas con discapacidad se ven enfrentadas a muchos obstáculos en la búsqueda de un lugar de trabajo, debido principalmente a las barreras sociales, y aquellas propias de su discapacidad. El desarrollo normativo en Colombia en torno a la discapacidad ha favorecido las oportunidades de integración social de esta población, y ha brindado beneficios al sector empresarial para que fomente su inclusión. Los programas de responsabilidad social empresarial ya están considerando los procesos de integración al mundo laboral de las personas con discapacidad. Este trabajo de investigación busca establecer los ajustes razonables de tipo ergonómico que se deben tener en cuenta para favorecer los procesos de inclusión laboral de personas con discapacidad intelectual leve en el área operativa de una empresa manufacturera de cables. Para ello, es fundamental el análisis del sistema ergonómico con el fin de identificar las posibles interacciones de cada uno de sus elementos (ser humano, objeto y espacio físico) y las barreras susceptibles de intervención para la aplicación de ajustes razonables.

\section{PALABRAS CLAVE}

Ajuste razonable, discapacidad intelectual, inclusión laboral, barreras y facilitadores.

\section{ABSTRACT}

Currently, disability is recognized as a fundamental human rights issue, since most people with disabilities are faced with many obstacles in their search for a work place, mainly due to social barriers, and those of their own disability. The regulatory development in Colombia around disability has favored opportunities for social integration of this population and provided benefits to the business sector that encourages their inclusion. Corporate social responsibility programs are already considering the processes of integration into the world of work of people with disabilities. This research work seeks s to establish reasonable ergonomic adjustments that should be taken into account to favor the processes of labor inclusion of people with intellectual disabilities in the operational area of a cable manufacturing company. For this, the analysis of the ergonomic system is fundamental, in order to identify the possible interactions of each of its elements (human being, object and physical space) and the barriers susceptible to intervention for the application of reasonable adjustments. 


\section{KEYWORDS}

Reasonable accommodation, intellectual disability, labor inclusion, barriers and facilitators.

\section{INTRODUCCIÓN}

La inclusión de Personas con Discapacidad (PcD) al mundo laboral, como área fundamental de la vida, es una necesidad planteada desde la integración social, apoyada por la legislación que se ha desarrollado en torno a las oportunidades que debe brindarse a esta población que sufre condiciones de desigualdad social (Velásquez, 2011). La igualdad de oportunidades y de trato es un principio fundamental de la justicia social, que hace parte de la labor de la OIT desde su fundación en 1919 (Miranda, 2018).

Históricamente se han generado barreras sociales que han estigmatizado a este grupo de personas en el ámbito empresarial y educativo provocando la reducción de las oportunidades en comparación con el resto de la población. Algunos gobiernos han desarrollado políticas públicas para mejorar esta situación de acuerdo con las directrices internacionales, sin embargo, se mantiene la desigualdad de tipo laboral en esta población (Ferro, 2010).

Si bien la promoción de la igualdad a través de políticas públicas ha mejorado la percepción con relación a las habilidades de las $\mathrm{PcD}$, muy pocas empresas en Colombia tienen una política laboral clara y concreta que abarque el tema de la contratación de personal discapacitado1. Algunos estudios han encontrado que en los puestos de trabajo de PcD intelectual el nivel de riesgo ergonómico es alto; en el estudio ergonómico hecho por ART Toll, doce de trece trabajadores de un área tienen una afección musculo esquelética de miembro superior, encontrando los siguientes diagnósticos por ecografía: tenosinovitis de flexores y extensores de mano $92 \%$, tendinitis del tendón largo de la cabeza del bíceps $70 \%$ y tendinitis de supraespinoso $31 \%$, sin que exista una relación con la edad y sexo, mostrando una asociación con las actividades del puesto de trabajo. El análisis del desarrollo de las competencias de empleabilidad muestra niveles altos, esto nos conduce a pensar que el proceso de supervisión y apoyo en el puesto de trabajo realizado por los Centros Especiales de Empleo (CEE) potencia este tipo de competencias (Laborda Molla, Jariot García, \& González Fernández, 2021).

Las PcD pueden tener un desempeño óptimo en el ámbito laboral (Vargas, Reyes, Pinzón, \& Hoz, 2017) con el apoyo y la formación adecuada, y desarrollar habilidades adaptativas que les permitan ser productivos en la sociedad, para lo cual las empresas deben realizar adecuaciones tanto en sus plantas físicas como en los procesos organizacionales según análisis ergonómicos de los puestos de trabajo. En la inclusión de $\mathrm{PcD}$ al trabajo esto es especialmente válido, fundamentalmente debido a que los puestos de trabajo en cada caso deben ser diseñados considerando sus características especiales e individuales, que muchas veces condicionan la integración del sujeto al trabajo (Zavala Briceño, 2019).

El método de evaluación de puestos de trabajo desarrollado por Pacto de Productividad permite diagnosticar facilitadores y barreras para la inclusión laboral de personas con discapacidad.

El objetivo del presente trabajo fue determinar los ajustes razonables de tipo ergonómico que deben ser considerados para favorecer la inclusión laboral de personas con discapacidad intelectual leve en el área de empaque de una empresa manufacturera.

\section{MATERIALES Y MÉTODO}

Estudio observacional, descriptivo. La población y la muestra se conformaron por 6 trabajadores. Los criterios de inclusión fueron: estar calificado con discapacidad intelectual leve y pertenecer al puesto de trabajo de empaque. El instrumento de recolección de información fue la Evaluación de puesto de trabajo del Pacto de Productividad, basado en la Convención 
Internacional de Derechos de las Personas con Discapacidad (Santacruz, Soto, Cortés, \& López, 2014). Los componentes y medios de validación del modelo fueron: 1. Movilización del sector empresarial, 2. Fortalecimiento técnico de instituciones de inclusión laboral, 3. Formación para el trabajo y 4. Articulación e incidencia en Política Pública. Se hizo análisis ergonómico de puesto de trabajo Ayudante de empaque, se desarrolló un diagnóstico de facilitadores y barreras para la inclusión laboral de personas con discapacidad en la empresa. Esto incluye la aplicación de la lista de chequeo para la evaluación del compromiso empresarial en el proceso de inclusión laboral de personas con discapacidad, la aplicación del formato de evaluación del proceso de inducción, y la aplicación de la matriz integral para el diagnóstico de barreras, todos documentos brindados en el documento antes mencionado.

\section{RESULTADOS}

\section{Organización del trabajo}

El puesto de trabajo definido para la inclusión laboral es Ayudante de empaque, este puesto hace parte del área de producción y pertenece al nivel operativo, no requiere experiencia o entrenamiento previo para el ingreso. Está diseñado para ser ejecutado por hombre mayor de 18 años con escolaridad bachiller. Esta labor está contratada a través de una empresa temporal a término fijo por un período de 7 meses. La jornada laboral es de 8 horas, en tres turnos rotativos 6 am a 2 pm, 2 pm a 10 pm, 10 pm a 6 am; con 20 min para el almuerzo o la cena de acuerdo con el turno. El jefe inmediato es el supervisor de producción.

El trabajador nuevo pasa por un proceso de inducción a la compañía y al puesto de trabajo. Es capacitado en riesgos laborales y acompaña a un operario en la planta durante 15 días en calidad de observador sin tener contacto con las máquinas.

\section{DESCRIPCIÓN DE LAS TAREAS}

Se observó que el puesto de trabajo requiere de tres tareas principales: sellar cajas, estibar y asear el puesto de trabajo, las dos primeras constituyen más de $80 \%$ del tiempo efectivo de trabajo. Para determinar la naturaleza de los ajustes razonables de carácter ergonómico, fue necesario comprender tanto la actividad como las tareas que se desarrollan, las cuales se describen a continuación:

\section{TAREAS}

Sellar cajas: El trabajador dirige la caja a través del sistema de rodillos hacía la encintadora donde se sella de manera automática. Dirige la caja hacia la báscula en donde el trabajador se encuentra y le pone un Tucker (Sticker con el peso de referencia), verifica que el peso corresponda al establecido en el Tucker, toma la caja con el Jumbo Flex y la traslada hasta la estiba. Las operaciones que componen en esta tarea son: operar mesas de rodillos y verificar los stickers que salen de la encintadora.

Estibar: El colaborador toma la caja desde la báscula haciendo uso del Jumbo Flex y la traslada hasta la estiba indicada según el tipo de referencia y procede a estibar. Asear: Al finalizar el turno barre los residuos de aserrín provenientes de las estibas, recoge el papel sobrante de los stickers y los ubica en los recipientes destinados a su disposición. Las operaciones son: barrer, recoger papel, disponer residuos.

\section{SISTEMA ERGONÓMICO}

La actividad de empaque es un sistema ergonómico tipo 5, toda vez que se genera interacción entre el trabajador, el espacio y varios objetos: sistema de rodillos, caja, encintadora, báscula, Tucker.

\section{EXIGENCIAS HUMANAS DEL PROCESO}

Para el desarrollo de la actividad de empaque el trabajador requiere las siguientes exigencias humanas Fisiológica

- Esfuerzo dinámico: Para desplazamiento constante de la báscula hacia la estiba y viceversa, uso de los brazos para sujetar el equipo que mueve las cargas 


\section{Sensorio-motor}

- Visual: para la ubicación espacial, reconocimiento de las máquinas, verificación de los sticker, identificación del estado de las cajas y las estibas.

- Auditivo: para diferenciar sonidos, sobre todo aquellos relacionados con los sistemas de emergencia y de las máquinas, se requiere discriminación y memoria auditiva.

- Discriminación visual de color: para identificar alarmas visuales, números $\mathrm{y}$ grafemas.

- Percepción de profundidad: para definir la estiba que se requiere de acuerdo con el tipo de caja.

- Integración bilateral: Para la manipulación de los elementos de trabajo, ejecución de movimientos disociados, simultáneos y alternados.

\section{Cognoscitivo}

- Resolución de problemas: capacidad para identificar y resolver con calma una situación de conflicto en el puesto de trabajo como una falla en la máquina o un represamiento de las cajas en la banda transportadora.

- Atención selectiva: para mantenerse en la actividad y ejecutarla sin distraerse por estímulos circundantes, atento al funcionamiento de las bandas y las básculas.

- Memoria de trabajo: para ubicar de las estibas y las cajas de una forma determinada de acuerdo con el instructivo establecido y a las referencias, recordar instrucciones que se brindan durante la ejecución de la actividad.

- Seguimiento de instrucciones: para recordar y ejecutar instrucciones compuestas por 2 o más comandos de sus jefes o compañeros de trabajo.

Tolerancia a la actividad: para iniciar y finalizar la actividad; tolerar el turno de trabajo.

\section{DIAGNÓSTICO DE BARRERAS Y FACILITADORES PARA LA INCLUSIÓN LABORAL}

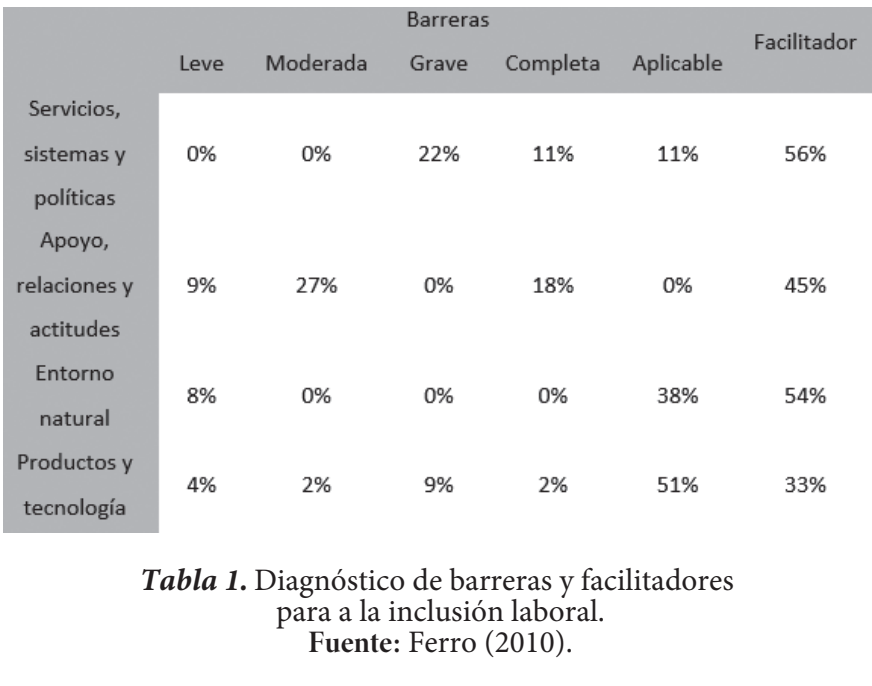

Para la identificación de barreras y facilitadores se aplicó la matriz del programa Pacto de Productividad y se obtuvo los siguientes resultados.

\section{PRODUCTOS Y TECNOLOGÍA}

La matriz evalúa 55 aspectos del componente productos y tecnología, encontrando que el 51\% de los aspectos que evalúa no aplican para el tipo de discapacidad intelectual, dichos factores hacen referencia a las interacciones con elementos de comunicación y desplazamiento, así como las ayudas (rampas, elevadores) para facilitar la comunicación de personas con limitación auditiva o física respectivamente; asimismo, se obtuvo un 33\% de facilitadores, es decir, aspectos que ayudarán a la inclusión en la compañía y al puesto de trabajo, entre estos aspectos se tienen: transporte proporcionado por la empresa para llegar a ella, dispositivos para 
comunicación visual (TV, video, paneles informativos), herramientas (Jumboflex, mesas elevadoras) para facilitar la ejecución de la labor, espacios de trabajo con zona de acceso libre de obstáculos, pisos antideslizantes y condiciones acústicas óptimas que facilitan la comunicación.

En cuanto a las barreras se obtuvo que se presenta un 9\% de barreras graves, es decir, que se deben intervenir a corto plazo, entre estas se tienen: no se dispone de elementos para la comunicación con dibujos, símbolos y escritura manual, pues todos los elementos proporcionados por la empresa se basan en la escritura, al asumirse que los trabajadores cuentan con un nivel de lectura y comprensión alto; por último, se observa que las barreras completas constituyen un $2 \%$ y se relacionan con la falta de sistemas luminosos o que hagan contraste con las paredes para identificar enchufes o interruptores, en este caso se relaciona con el panel de control para el accionamiento de las mesas de rodillos, en las cuales se encuentra que todos los botones son de igual color para accionar todas y cada una de las líneas, lo que puede llegar a generar confusión en el trabajador.

\section{ENTORNO NATURAL Y CAMBIOS EN EL ENTORNO DERIVADOS DE LA ACTIVIDAD HUMANA}

Este componente evalúa 13 aspectos de los cuales el $54 \%$ constituyen un facilitador, pues al evaluarse temas como ruido o iluminación se encuentra que están dentro de los límites permisibles, facilitando la comunicación entre los individuos.

El 38\% de los aspectos no aplican para el tipo de discapacidad y solo se cuenta con una barrera leve, que hace referencia a que se dispone de información visual luminosa con contraste de color apropiado para ver escaleras, puertas, alarmas, rutas o avisos relacionados con la labor. Esta barrera debe ser intervenida a largo plazo y constituye el $8 \%$. Aunque la empresa cuenta con esta tecnología no se observa en todas las áreas de la compañía.

\section{APOYO, RELACIONES Y ACTITUDES}

En este aspecto se evaluaron 11 factores, de los cuales el $45 \%$ son facilitadores pues existe compromiso desde la gerencia para la inclusión de personas en condición de discapacidad, brindando los recursos suficientes para mejorar la calidad de vida de los trabajadores, creando políticas y sistemas de bienestar.

Debido a que se desconoce la percepción de los trabajadores frente a la inclusión de discapacidad, se han creado barreras completas, es decir, de intervención inmediata las cuales constituyen un $18 \%$.

\section{SERVICIOS, SISTEMAS LABORALES Y DE EMPLEO}

En este aspecto se evaluaron 9 componentes, de los cuales el 56\% son facilitadores, pues la dirección está consciente de los ajustes que deben realizar para lograr la inclusión de las personas en condición de discapacidad. Para este fin se han creado políticas y estrategias para dar a conocer entre los trabajadores, buscando mejorar la cultura de la inclusión y disminuir los niveles de exclusión; asimismo se encuentra en proceso de creación de alianzas estratégicas con entidades que promuevan la formación y habilitación de personas con discapacidad para traerlas al proceso productivo.

En este punto se observa un 11\% de barreras completas relacionadas con el apoyo de la ARL para la integración de personas con discapacidad, aspecto que cobra gran importancia, pues se tendrá a una persona expuesta a riesgos que pueden conllevar a accidentes o enfermedades laborales y se deben diseñar estrategias de acuerdo con su condición que promuevan su autocuidado en el ambiente laboral.

\section{COMPROMISO EMPRESARIAL}

En cuanto al compromiso gerencial se obtuvo un resultado de 13/20 puntos lo que indica que la empresa cuenta con un compromiso excelente, por cuanto la 
iniciativa de incluir personas con discapacidad surge desde Gestión Humana, lo cual habla del compromiso que tiene la empresa para este proceso, ya que se gestionarán los elementos necesarios para alcanzar el objetivo previsto; así mismo se determina que la percepción de los directivos y mandos medios para la inclusión es favorable, lo que permitirá crear políticas de inclusión y permearán la cultura organizacional, sobre todo si se empiezan a generar cambios desde las cabezas visibles de la organización.

Finalmente, el compromiso gerencial se hace visible en el acuerdo de realizar los ajustes razonables a que haya lugar para lograr la inclusión de personas con discapacidad.

De acuerdo con los resultados obtenidos y el análisis realizado se consideran los siguientes ajustes razonables:

\section{AJUSTES OBJETOS/MÁQUINAS}

- $\quad$ Estibas: Para la identificación de la referencia de estiba adecuada acorde con el tipo de producto a estibar, se recomienda la identificación por colores de estas, según su referencia, con el fin de involucrar la percepción visual de colores y reducir las exigencias para la ubicación espacial e identificación de dimensiones.

- $\quad$ Panel de control de las líneas: Se recomienda mejorar la identificación de los botones ubicados en el panel de control para operar el funcionamiento de las mesas de rodillos, de tal manera que se permita con facilidad identificar a qué botón corresponde cada mesa, a través de un sistema con números o colores en el panel, que se relacione con la señalización respectiva ubicada sobre la línea.

- Instructivos: Los documentos que contienen las instrucciones del proceso de estibado deben incluir ayudas visuales: imágenes, colores, que permitan la identificación de las estibas, el número de niveles de estibado, cantidad de cajas por nivel, de acuerdo con la referencia del producto a estibar.

\section{AJUSTES POLÍTICAS, ORGANIZACIÓN Y SELECCIÓN}

- Creación de una política de inclusión laboral para personas con discapacidad.

Establecer alianzas estratégicas con fundaciones

- Diseñar e implementar un programa de inclusión laboral para personas con discapacidad.

- Asignar dentro de los responsables del sistema de gestión de seguridad y salud laboral, el seguimiento y la verificación del cumplimiento de las acciones establecidas en el programa de inclusión laboral.

Construcción de un perfil ocupacional con énfasis en procesos perceptivos $y$ senso-perceptivos.

Implementar la realización de pruebas psicológicas para validar el cumplimiento del perfil

- Contratación. Se recomienda realizar ajustes al contrato laboral, especificando la condición de persona con discapacidad y la vinculación directa por contrato laboral.

\section{AJUSTES A LA INDUCCIÓN}

- Se recomienda que los procesos sean prácticos (didácticos).

- Reconocimiento de los diferentes espacios a través de recorridos. 
- Contacto inicial de los elementos del puesto de trabajo.

- Implementación de un programa padrino para el acompañamiento en el puesto de trabajo.

\section{AJUSTES A LA FORMACIÓN}

- Capacitación que incluya componentes auditivos, visuales y prácticos.

- $\quad$ Procesos de sensibilización a la comunidad de la empresa frente a la discapacidad.

- Realizar formación específica de los trabajadores que harán las funciones de padrino.

\section{DISCUSIÓN}

En el puesto de trabajo de Ayudante de empaque se observa que las exigencias cognoscitivas son las de mayor impacto; la puesta en práctica de estas le permite al trabajador interactuar de manera adecuada con los objetos y el espacio físico dando respuesta a las exigencias de productividad que se plantean para el puesto.

Por otro lado, los componentes motores juegan un papel importante, sin embargo, con la ejecución frecuente de la tarea, esta se convierte en una praxia siendo casi mecánica su ejecución (tomar la caja, accionar el jumboflex, ubicarlo en la estiba), sin embargo, debe realizar un análisis previo para tomar la decisión de dónde y cómo ubicar la estiba, decisión que se toma gracias a la puesta en marcha de todos los aspectos cognitivos descritos en el apartado anterior.

En este orden de ideas y teniendo en cuenta que se buscará la inclusión de $\mathrm{PcD}$ intelectual leve, se considera necesario centrar los esfuerzos en realizar ajustes que le permitan al trabajador responder de manera adaptativa a las exigencias de la tarea, gracias a la facilidad que tiene para percibir procesos y comprender la información que se le brinda.
Por esta razón los procesos de inclusión laboral para PcD deben desarrollarse considerando todos los posibles escenarios en los cuales pueda interactuar el individuo al interior de la organización, con el fin de que se garanticen las mismas oportunidades que a las demás personas.

Si bien, muchos trabajos coinciden en resaltar el desarrollo que han tenido los países en torno a sus políticas relacionadas con la discapacidad, aun no existe la cultura de contratación de esta población por parte de las empresas. Para el caso de Colombia no es diferente esta realidad, y se requieren estudios para medir la percepción de la inclusión laboral de PcD. Del mismo modo, no existe suficiente literatura con relación a los ajustes de tipo ergonómico necesarios para los procesos de inclusión laboral de $\mathrm{PcD}$.

El aporte de la ergonomía, como disciplina, para el análisis de las interacciones que ocurren en cada uno de los elementos del sistema ergonómico y de la influencia de su contexto, es fundamental para definir los focos de intervención y así favorecer una completa y adecuada integración de las PcD.

Realizar un diagnóstico de barreras contribuye a la identificación y priorización de los factores susceptibles de modificación en el corto, mediano y largo plazo, a través de la práctica de ajustes razonables. La matriz de diagnóstico de barreras desarrollada por el programa pacto de productividad, se convierte en un elemento de gran utilidad para la identificación de los factores externos que puedan influir positiva y negativamente en el desempeño del individuo con discapacidad.

\section{CONCLUSIÓN}

Se requieren más estudios con relación a la discapacidad en Colombia y la percepción por parte de la población en general y de los empleadores, y estudios que permitan conocer los resultados obtenidos por parte de aquellas empresas que hayan considerado e implementado ajustes de tipo ergonómico dentro de sus programas de inclusión laboral para $\mathrm{PcD}$. 
Es importante que por parte de los gobiernos se desarrollen nuevas estrategias para brindar apoyo a las empresas para estructurar sus políticas y ejecutar programas de inclusión laboral. La asesoría por parte de las Administradoras de Riesgos Laborales es fundamental para que finalmente despeguen estas iniciativas a nivel nacional, como también la participación de equipos multidisciplinarios que incluyan terapeutas ocupacionales, fisioterapeutas, médicos con formación en ergonomía, con el fin de orientar las adaptaciones o ajustes al sistema ergonómico durante el proceso de inclusión laboral de PcD.

\section{REFERENCIAS BIBLIOGRÁFICAS}

Agudelo, M. L., Casas, E. L., Tovar, A. y Villadiego, E. personas con discapacidad en el ámbito laboral (Trabajo de grado). Universidad Sergio Arboleda, Bogotá, Colombia. https://repository.usergioarboleda.edu.co/ handle/11232/1005

Fidan, A. , Cihan, H. , \& Özbey, F. (2014). An Important Component in Successful Inclusion Practices: Instructional Adaptations. Procedia - Social and Behavioral Sciences, 116 . doi: 10.1016/j.sbspro.2014.01.1045

Anizam Mohamed Yusof, Manisah Mohd Ali \& Amla Mohd Salleh, (2014). Employability of Vocational School Leavers with Disability. Procedia: Social and Behavioral Science 112, 1064-1069.

Pupiales Rueda, B.E., \& Córdoba Andrade, L. (2016). La inclusión laboral de personas con discapacidad: Un estudio etnográfico en cinco comunidades autónomas de España. Archivos de Medicina (Col), 16(2), 279-289. https://www.redalyc.org/ articulo.oa? $\mathrm{id}=273849945007$
Burge, P., Lysaght, R., \& Eastern, S. (2007). Public views on employment of people with intellectual disabilities. Journal of Vocational Rehabilitation, 26, 29-37.

Correa-Montoya, L. (2016). Disability and social inclusion in colombia. Saldarriaga-Concha Foundation Alternative Report to the Committee on the Rights of Persons with Disabilities

de Guimarães, B. M., Martins, L. B., \& Junior, B. B. (2015). Workplace adaptation to people with disabilities at construction industry in Brazil. Work (Reading, Mass.), 50(4), 575-584. https://doi.org/10.3233/WOR-131813

Guerrero, C. (2015). Experiencias y prácticas de Empleo con Apoyo llevadas a cabo por la Federación de Asociaciones en favor de las Personas con Discapacidad Intelectual de la Región de Murcia. Inclusión laboral de personas con discapacidad en Murcia España. Enlace en Red, 23, 32-34.

Ferro, F. E. (2010). Estudio diagnóstico sobre barreras para la inclusión laboral. Pacto de Productividad.

Fernández, A. (2013). El nivel de paro general, los prejuicios sociales y empresariales, la baja cualificación profesional de las personas con discapacidad, falta de adaptación de los puestos de trabajo. Factores que dificultan la inserción laboral de las personas con discapacidad.

García Solano, H., Camelo Pérez, F., Rodríguez Pinto, L.L. (2017) El Diseño como Facilitador de la Inclusión Laboral de Personas en Condición de Discapacidad Física. Caso Almacenes Paraíso S.A. Revista Encuentros, 15(02). http://dx.doi.org/10.15665/re.v15i2.663 
Laborda Molla, C., Jariot García, M., \& González González Fernández, H. (2020). Calidad de vida y competencias de empleabilidad en personas trabajadoras en centros especiales de empleo. Educación XX1, 24(1). doi:https://doi.org/10.5944/educxx1.26570

MacDonald-Wilson, K., Fabian, E., \& Dong, S. (2008). Best practices in developing reasonable accommodations in the workplace: Findings based on the research literature. The Rehabilitation Professional, 16(4), 221-232.

Mareño Sempertegui, M. (2015). Inclusión. laboral de personas catalogadas como discapacitadas. Algunas reflexiones para un debate necesario. Trabajo y Sociedad, (25), 405-442. https://www.redalyc.org/articulo. oa? id=387341101023

Villavicencio Miranda, L. (2018). Justicia social y el principio de igualdad. HYBRIS. Revista de Filosofía, 9, 43-74

Neves-Silva, P., Prais, Gomes, F. \& Silveira, A.M. (2015). The inclusion of disabled persons in the labor market in Belo horizonte, Brazil: scenario and perspective. Ciência \& Saúde Coletiva, 20(8), 2549-2558. https://doi.org /10.1590/1413-81232015208.17802014

Organización Internacional del Trabajo. (2015). Estrategia y plan de acción para la inclusión de la discapacidad 2014-17: un doble enfoque de acciones transversales y específicas para las personas con discapacidad. OMS.

Organización Internacional de Trabajo. (2017). Fomentando la diversidad y la inclusión mediante los ajustes en el lugar de trabajo, una guía práctica. OMS.
Pallisera, M., Fullana, J., \& Vila, M. (2005). La inserción laboral de personas con discapacidad. Desarrollo de tres investigaciones acerca de los factores favorecedores de los procesos de inserción. Revista de Investigación Educativa, 23(2), 295-313. https://revistas.um.es/rie/article/view/97721

Ramalho-Pires de Almeida, M. Á., Ábalos-Medina, G. M., Villaverde-Gutiérrez, C., Gomes-de Lucena, N. M., Ferreira-Tomaz, A., \& Perez-Marmol, J. M. (2019). Effects of an ergonomic program on the quality of life and work performance of university staff with physical disabilities: A clinical trial with three-month follow-up. Disability and health journal, 12(1), 58-64. https://doi. org/10.1016/j.dhjo.2018.07.002

Randt, N. (2016). CommonKnowledge Emerging Practice CATs OT Critically Appraised Topics 2011 Social Inclusion for People with Intellectual Disabilities.

Santacruz, M. Z., Soto, J. A., Cortés, O. L., \& López, G. C. (2014). Formato guía de aspectos genéricos de los análisis de puestos de trabajo. Pacto de Productividad.

Seoane, B. (2017). Deficiencia, Minusvalía y Discapacidad. Guía para la inclusión de personas con discapacidad en el ámbito laboral.

Servicio Nacional de Discapacidad. (2014). Estudio de inclusión laboral de personas en situación de discapacidad en el estado de Chile. Ministerio de Desarrollo Social Gobierno de Chile.

Sherlaw, W. \& Hudebine, H. (2015). The United Nations Convention on the rights of persons with disabilities: Opportunities and tensions within the social inclusion and participation of persons with disabilities. Alter, 9(1), 9-21. 
Vargas, M. L., Reyes, E. L., Pinzón, A. T., \& Hoz, E. V. (2017). Guía para la inclusión de personas con discapacidad en el ámbito laboral. Universidad Sergio Arboleda.

Velasco-Jáuregui, L.C. (2013). El proceso de inclusión laboral de las personas con discapacidad. Un estudio comparativo entre España y México (Tesis doctoral, Doctorado en Estudios Científico Sociales). Tlaquepaque, Jalisco.

Vidal, R. y Vilos, V. (2012). Elaboración de un modelo para la inserción laboral de personas con discapacidad intelectual en la Región del Maule. Universidad Católica del Maule, Talca-Chile. Manuscrito no publicado.

Vidal, R., Cornejo, C. y Arroyo, M. L. (2013) La inserción laboral de personas con discapacidad intelectual en Chile. Convergencia Educativa, 2, 93-102.

Velásquez, E. D. (2011). Estratificación y desigualdad por motivo de discapacidad. Intersticios. Revista sociológica de pensamiento crítico, 5(1), 157-170.

Ke, X. y Liu, J. (2017). Discapacidad intelectual. Trastornos del desarrollo. https://iacapap.org/ content/uploads/C.1-Discapacidad-Intelectual-SP ANISH-2018.pdf

Zavala Briceño, D. (2019). Métodos ergonómicos de inclusión de personas en situación de discapacidad al trabajo. Ergonomía, Investigación y Desarrollo, 1(1), 158 - 170. https:// revistasacademicas.udec.cl/index.php/Ergonomia _Investigacion/article/view/1289

\section{AUTORES}

Diana Yulieth Castañeda Zapata: terapeuta Ocupacional, Universidad del Valle. Correo: dianacastaneda.to@gmail.com

Jessica María Carvajal Pardo: fisioterapeuta, Universidad María Cano. Correo: ftjekarvajal@gmail.com

Adrián Augusto Leal Aranguren: médico, Universidad Libre. Correo: adrianleal11@gmail.com

Luz América Martínez Álvarez: Diseñadora Industrial, Especialista en Higiene y Seguridad Industrial, Magíster en Ergonomía, Magíster en Dirección de proyectos. Institución Universitaria Antonio José Camacho. Correo: lamericamartinez@admon.uniajc.edu.co

Cecilia Andrea Ordóñez Hernández: fisioterapeuta, Magíster en Salud Ocupacional, Doctora en Ciencias de la Salud en el Trabajo. Docente Universidad del Libre Cali. Correo: andrea.ordonezh@unilibre.edu.co 